\title{
Trabajo social en el campo socioambiental: discusiones, énfasis y desafíos a partir de la Conferencia Mundial de Trabajo Social 2018 ${ }^{1}$
}

\author{
Elia Sepúlveda Hernández ${ }^{2}$
}

\begin{abstract}
RESUMEN
Desdelas diversas problemáticas y emergencias socioambientales que enfrenta el mundo contemporáneo, este artículo busca conocer el debate que, respecto de este tema, se desarrolló en Dublín, en el marco de la Conferencia Mundial Conjunta sobre Trabajo Social, Educación y Desarrollo Social 2018. Se realizó una revisión y análisis del Abstract Book de la conferencia, específicamente de aquellas que conformaron el tópico "Vinculación ambiental y desarrollo sostenible". Los resultados describen el origen geográfico de las ponencias, el tipo de ponencias enviadas y los temas específicos que se abordan. Si bien se reconocen brechas en cuanto a la exploración, teorización o aplicación del trabajo social ambiental como subcampo disciplinar, existe un acumulado de investigaciones, experiencias y herramientas de alto valor, que se expresa en la diversidad y riqueza del debate académico expuesto en la conferencia.

Palabras clave: debate académico, problemas socioambientales, cambio ambiental global, trabajo social
\end{abstract}

\section{Social Work in the Socio-Environmental Field: Discussions, Emphasis and Challenges from the World Conference on Social Work 2018.}

\author{
ABSTRACT
}

Based on the different socio-environmental issues and emergencies faced by the contemporary world, this article reports on the debate held on this topic in Dublin within the framework of the Joint World Conference on Social Work, Education and Social Development 2018. The conference's Abstract Book was reviewed and analyzed, specifically those papers related to the theme of "Environmental and Community Sustainability." The results describe the geographical origins of the papers, the type of papers submitted and the

Artículo recibido el 13/09/2018. Artículo aprobado el 23 de diciembre de 2018.

Chilena. Trabajadora social. Becaria CONICYT Programa Doctorado en el Extranjero. E-mail: eliasepulvedah@gmail.com 
Trabajo social en el campo socioambiental: discusiones, énfasis y desafíos a partir de la Conferencia Mundial de Trabajo Social 2018 /Sepúlveda

specific topics they address. Despite gaps in the exploration, theorization or application of social work as a disciplinary subfield, there are a number of research studies, experiences and high-value tools expressed in the diversity and richness of the academic debate generated by the conference.

Keywords: academic debate, socio-environmental problems, global environmental change, social work

\section{Trabalho social no campo socioambiental: discussões, ênfases e desafios a partir da Conferência Mundial de Trabalho Social 2018}

Desde as diversas problemáticas e emergências socioambientais que enfrenta o mundo contemporâneo, este artigo busca conhecer o debate que, a respeito desta matéria, foi desenvolvido em Dublin no quadro da Conferência Mundial Conjunta sobre Trabalho Social, Educação e Desenvolvimento Social 2018. Foi realizada uma revisão e análise do Abstract Book da conferência, nomeadamente daquelas apresentações que conformaram o tópico "Vinculação ambiental e desenvolvimento sustentável". Os resultados descrevem a origem geográfica das palestras, o tipo de palestras enviadas e os temas específicos que se tratam. Embora se reconheçam lacunas quanto à exploração, teorização ou aplicação do trabalho social ambiental como subcampo disciplinar, existe uma quantidade importante de pesquisas, experiências e ferramentas de alto valor, que se expressa na diversidade e riqueza do debate acadêmico exposto na conferência.

Palavras-chave: debate acadêmico, problemas socioambientais, mudança ambiental global, trabalho social

\section{Introducción}

Entre el 4 y el 7 de julio del año 2018, en la ciudad de Dublín, Irlanda, se desarrolló una nueva versión de la Conferencia Mundial Conjunta sobre Trabajo Social, Educación y Desarrollo Social 2018 (SWSD 2018): "Social Work, Education and Social Development Environmental and community sustainability | Human solutions in evolving societies".

Esta versión del SWSD tuvo como foco principal el tercer pilar de la Agenda Global para el Trabajo Social, o sea, trabajar hacia la sostenibilidad del medio ambiente. La relación entre la sociedad y la naturaleza enfrenta un punto de inflexión, en el que el mode- 
lo de desarrollo económico capitalista-extractivista choca con los ritmos y ciclos de la biosfera y los sistemas ecológicos (Naredo, 2006; Martínez Alier, 2001). Dicha situación ha provocado un aumento significativo en los impactos sociales y económicos de los desastres y conflictos medioambientales (UNISDR, 2015).

El Centre for Research on the Epidemiology of Disaster (CRED), menciona que entre 1998 y 2017 los desastres naturales asociados al clima y eventos geofísicos mataron un total de 1,3 millones de personas en el mundo, y dejaron 4,4 mil millones de heridos, desplazados, sin hogar o con necesidad de asistencia en emergencia. El 91\% de estos desastres se han producido por eventos metereológicos extremos, como inundaciones, tormentas, sequías y olas de calor (Wallemacq, Below y McLean, 2018).

El EM-DAT The International Disaster database -la base de datos de desastres naturales más completa del mundo-, subraya la gran desigualdad entre las naciones respecto de la carga que implica un desastre. Los países más pobres asumen el mayor costo de daños a largo plazo y pérdida de vidas humanas, lo que evidencia el cruce entre desastre, conflicto socioambiental y pobreza. En este sentido, y tal como se afirma en el Marco de Sendai para la reducción de riesgo de desastres 2015-2030, reducir la pobreza y avanzar hacia mayores estándares de justicia e igualdad en el mundo requiere poner atención en las estrategias para hacer frente a problemáticas como el cambio climático o el cambio ambiental global en su conjunto.

Haciendo eco de esta realidad, la SWSD 2018 incorpora la cuestión ambiental de manera central dentro de la discusión disciplinar, lo que ya había sido sugerido dentro de las conclusiones del SWSD del año 2016 celebrado en Seúl, y en la Agenda Global para el Trabajo Social.

Dicha agenda se consolida como un esfuerzo mundial de cooperación y unidad que, desde el año 2010, instala pilares, prioridades y mensaje comunes para fortalecer las contribuciones de la profesión a las políticas sociales locales y globales. A través de la voz 
Trabajo social en el campo socioambiental: discusiones, énfasis y desafíos a partir de la Conferencia Mundial de Trabajo Social 2018 /Sepúlveda

colectiva y compartida de diferentes organizaciones internacionales de trabajo social, la agenda prioriza problemáticas sociales de alta relevancia y complejidad contemporánea, entre ellas, aspectos relacionados con los efectos sociales del cambio climático, los desastres naturales, los contaminantes y la guerra. Dichos efectos van en desmedro de la salud y el bienestar de personas afectadas por grandes desigualdades y entornos insostenibles (Truell y Jones, 2017).

En consecuencia, desde la Asociación Internacional de Escuelas de Trabajo Social (IASSW), el Consejo Internacional de Bienestar Social (ICSW) y la Federación Internacional de Trabajadores Sociales (IFSW) se reconoce la emergencia de esta problemática. Además, se asume como un fenómeno que se vincula de manera directa con el quehacer profesional del trabajo social. Un quehacer que está marcado, entre otras cosas, por la atención a urgencias sociales, crisis y desastres tanto individuales como comunitarios.

Si bien en los últimos quince años las publicaciones de trabajo social relacionadas con temas ambientales se han triplicado, lo que demuestra un cambio sustancial en la atención al tema, aún persisten brechas disciplinares relacionadas con:

1) Falta de contenido ambiental en la enseñanza de la profesión. La alfabetización ambiental en trabajo social, por ejemplo, no es mejor que en la población promedio (Miller y Hayward, 2014).

2) La inmersión de la profesión en un contexto paradigmático de tipo antropocéntrico, capitalista y mecanicista, en el que la naturaleza se define como un recurso económico.

3) La dificultad para definir lo que implica el "trabajo social ambiental". Existen diversas interpretaciones, variedad de términos y descripciones que causan confusión y dificultades en la traducción a la práctica (Ramsay y Boddy, 2017).

Frente a ello, y para aportar al desarrollo disciplinar de la cuestión socioambiental, este artículo realiza una revisión documental con fuente secundaria, a partir de la discusión y reflexión académica que se desarrolló durante la última conferencia mundial de Tra- 
bajo Social SWSD 2018 en relación con el tema de la vinculación entre sociedad y naturaleza.

\section{Método de la revisión documental}

La revisión documental se realizó orientada por la siguiente pregunta de análisis: En relación con el desarrollo sostenible y al fenómeno de cambio ambiental global, ¿alrededor de qué temáticas y ámbitos de actuación profesional se desarrolló la discusión disciplinar de la SWSD 2018? Para responder a dicha pregunta se realizó una revisión documental cuya fuente fue el Abstract Book de la Conferencia Mundial Conjunta sobre Trabajo Social, Educación y Desarrollo Social 2018 (IASSW, ICSW y IFSW, 2018).

La SWSD 2018 organizó la Conferencia sobre la base de quince tópicos. De ellos, el tópico 1 aborda cuestiones relacionadas con vinculación ambiental y desarrollo sostenible, y el tópico 2 aborda el papel de trabajadores y trabajadoras sociales en el cumplimiento de los objetivos de Desarrollo Sostenible de la ONU. Específicamente, se analizó el contenido de los resúmenes de las ponencias que componen el "Tópico 1 de la SWSD 2018: Vinculación ambiental y desarrollo sostenible". Las personas organizadoras del evento agruparon en este tópico a las contribuciones relacionadas con temas socioambientales.

Dichos resúmenes se analizaron en función de los siguientes objetivos:

1. Determinar el origen geográfico de las ponencias, para así detectar la predominancia de países y áreas continentales presentes en la Conferencia respecto de este tópico en particular.

2. Conocer los tipos de comunicaciones enviadas. En específico, si se referían a resultados de investigación, a hallazgos que surgen desde la intervención o reflexiones teóricas disciplinares o multidisciplinarias ${ }^{3}$.

Los resultados de investigación serán entendidos como la socialización de un conocimiento que surge desde la aplicación del método científico. Por otra parte, 
Trabajo social en el campo socioambiental: discusiones, énfasis y desafíos a partir de la Conferencia Mundial de Trabajo Social 2018 /Sepúlveda

3. Determinar categorías o áreas temáticas que prevalecen en la discusión. Para cada una de ellas, identificar temas o problemáticas específicas que componen dicha discusión.

A partir del Abstract Book de la SWSD 2018 se identificaron 84 resúmenes de ponencias. El contenido de cada resumen se revisó y analizó en función de tres elementos de clasificación: a) país y zona continental de origen, b) categoría o temática central que aborda y c) tema específico o problemática a la que se refiere. Para analizar la información se utilizó el software Nvivo.

Respecto de la clasificación de categorías centrales detectadas (objetivo 3), es importante mencionar que dichas categorías no fueron predeterminadas, sino que se infieren a partir de la lectura de los resúmenes y el posterior análisis realizado por los autores del artículo. Además, se tuvieron en cuenta criterios como palabras clave y título del resumen, así como también el énfasis temático o conceptual en el contenido del texto.

\section{Resultados}

Los resultados se han organizado en función de los objetivos de la revisión documental. Comienza con la identificación del origen geográfico (continente) de los resúmenes de ponencia y luego se describen los tipos de resúmenes, según si se refieren a procesos de investigación, intervención o reflexión disciplinar. A continuación se mencionan las categorías centrales detectadas y los temas específicos por cada categoría.

A través de estos resultados se pretende entregar una descripción actualizada de algunos elementos centrales que componen la discusión del trabajo social, en relación con el desarrollo sostenible y la vinculación sociedad/naturaleza

los hallazgos desde la intervención se refieren a la entrega de resultados, descripción o análisis de procesos y prácticas de intervención social. Por último, la reflexión disciplinar se entenderá como una abstracción analítica personal a partir de insumos teóricos asociados al trabajo social o derivados de otras disciplinas. 


\section{a) Origen de las ponencias}

Para determinar el origen de las ponencias, se consideró la afiliación institucional declarada por las y los autores. Por tratarse de un evento académico, las universidades fueron las principales organizaciones mencionadas. También, aunque en menor medida, la afiliación se refería a organizaciones públicas o privadas que ejecutan proyectos o programas sociales con componente socioambiental.

De las 84 ponencias analizadas, 7 no mencionan su origen, por lo tanto no fueron consideradas en el conteo. Hay ponencias en modalidad "simposio" en las que existe más de un país involucrado.

En la figura 1 se presenta un mapa con la distribución de las ponencias por continente. En el caso de América latina y el Caribe se contabilizó dentro de esta zona geográfica a México y Barbados.

Figura 1. Distribución de las ponencias por zonas continentales

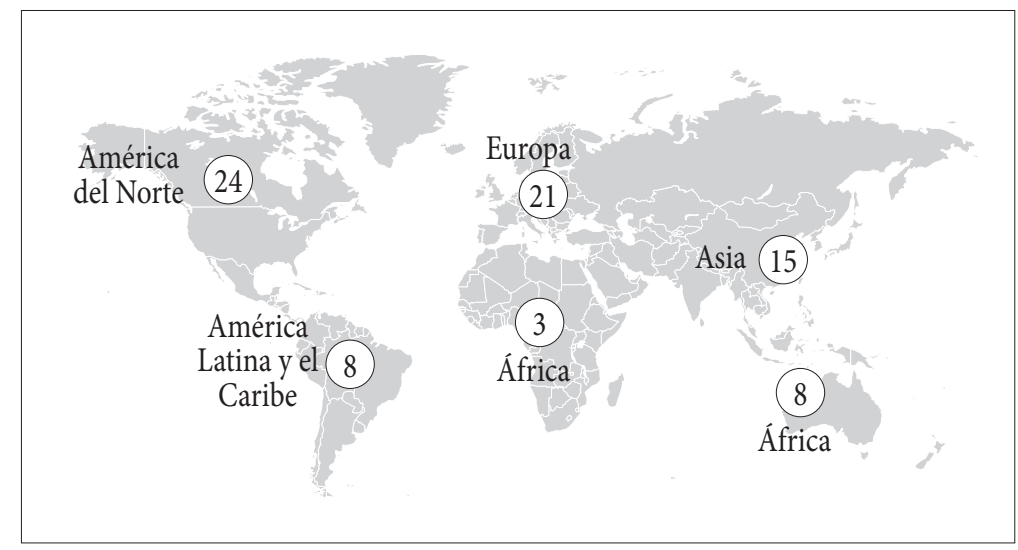

Fuente: elaboración propia.

Como es común en los procesos de producción científica, los países más ricos lideran la discusión académica. Estados Unidos es el país con mayor cantidad de resúmenes presentados, 18 en total. Le sigue China con 7 y Australia, Canadá e India con 6 resúmenes cada uno.

En el caso de América latina y el Caribe, Brasil concentra la presencia de la región a través de 5 resúmenes. A ello se suma un resumen de México, uno de Colombia y uno de Barbados. 
Trabajo social en el campo socioambiental: discusiones, énfasis y desafíos a partir de la Conferencia Mundial de Trabajo Social 2018 /Sepúlveda

En el caso de Europa, Reino Unido (5 resúmenes), Finlandia y Suecia (4 resúmenes cada uno) son los países que mayor cantidad de ponencias envían al Congreso. En el caso de Asia, a la presencia de China e India se suman países como Taiwán y Turquía con una ponencia cada uno.

En el caso de África, Sudáfrica envía resúmenes a través de académicos vinculados a las universidades de Pretoria y Limpopo. Dicho continente es la zona con menor cantidad de resúmenes enviados. En Oceanía, Australia presenta 6 resúmenes y Nueva Zelanda 2 y entre ambos países suman la misma cantidad de ponencias que la región latinoamericana.

Amplias zonas del mundo, como es el caso de Oriente Medio, la zona andina o austral de América del Sur y Rusia -entre otros- no tienen envío de resúmenes.

\section{b) Tipo de resumen}

Tal como se observa en la figura 2, hay una prevalencia de resúmenes que surgen como resultado de investigaciones y solo un 13\% se refiere a hallazgos o relatos de experiencias de intervención social directa. Estas últimas aluden principalmente a proyectos locales de trabajo ecosocial o trabajo social verde, que son destacados por su aporte a la construcción de entornos más sustentables.

Figura 2: Tipos de resúmenes.

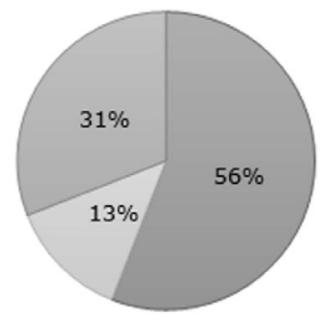

$\square$ Resultado de investigación

$\square$ Hallazgos desde la intervención social

$\square$ Reflexión disciplinar o multidisciplinaria

Fuente: Elaboración propia. 
Las investigaciones que se presentan surgen principalmente a partir del trabajo de estudiantes de posgrado o como parte de grupos o líneas de investigación de las universidades. La mayoría de los estudios utilizan metodologías de prevalencia cualitativa. La entrevista es la técnica más utilizada.

También se mencionan diseños de investigación referidos a estudios de casos y análisis sistemático de la teoría, este último para conocer la producción científica respecto de temas específicos. Un ejemplo es un análisis sistemático que utilizó la estructura de la declaración PRISMA 2009 (Preferred Reporting Items for Systematic reviews andMeta-Analyses) para estudiar la influencia del cambio ambiental global en la salud mental de pueblos indígenas.

Otro ejemplo es un estudio cienciométrico desarrollado en conjunto por investigadoras de la Universidad de Michigan, Chicago y Florida (EE.UU.), respecto de las tendencias de la literatura de trabajo social sobre temas ambientales.

Destacan las investigaciones de corte colaborativo, que considera el trabajo conjunto entre varias universidades, además de socios gubernamentales y comunitarios. Un ejemplo es la asociación "Rebuilding Lives Post-Disaster", que reúne a investigadores de trabajo social para estudiar la recuperación a largo plazo de desastres en Canadá, Estados Unidos, Australia, India, Pakistán y Taiwán.

A ello se suma la iniciativa desarrollada en el marco del Global Partnership for Transformative Social Work, que se desarrolló en Inglaterra durante 2017. En dicha instancia, trabajadores sociales de Australia, Finlandia, Alemania, Suecia y los Estados Unidos participaron en el taller "Climate change and the (lack of) future/intergenerational ethics in social work" y dialogaron sobre sus preocupaciones y luchas respecto del cambio climático. Este taller se replicó en el Congreso Mundial de Dublín 2018, con el fin de ampliar las redes disciplinares hacia un desarrollo sustentable. 
Trabajo social en el campo socioambiental: discusiones, énfasis y desafíos a partir de la Conferencia Mundial de Trabajo Social 2018 /Sepúlveda

Los resúmenes de ponencias de tipo reflexión disciplinar abordan asuntos relacionados con el papel del trabajo social dentro de la cuestión socioambiental. Por ejemplo, la incorporación de la temática dentro de los planes de estudio de formación en trabajo social o el papel del trabajo social en la construcción de comunidades más sustentables.

Son reflexiones que problematizan la crisis ambiental desde las dimensiones económicas (capitalismo), políticas y culturales en juego. Junto con ello, incorporan conceptos teóricos pertinentes para comprender y pensar la crisis ambiental y la construcción de comunidades más sustentables.

Un ejemplo de ello son tres ponencias que se refieren al concepto de "Antropoceno". Destaca el trabajo de A. Whiteford, de Plymouth University, Reino Unido. La ponencia tiene como hito un viaje en velero desde Dublín a Reino Unido, tripulado por el autor, su familia y colegas de trabajo social. A partir de esa vivencia, menciona que es posible construir nuevos relatos respecto de la sostenibilidad, el mundo material (desde la comprensión de lo biológico hasta la experiencia humana) y el concepto mismo de "Antropoceno".

Las tres ponencias que abordan dicho concepto concuerdan en que es un insumo teórico que entrega elementos útiles para análisis críticos respecto de la relación contemporánea entre naturaleza, cultura y sociedad.

\section{c)Distribución de los resúmenes de ponencia según las categorías detectadas}

En la figura 3 se presentan las cinco categorías detectadas, que permiten clasificar las tendencias temáticas respecto del contenido de los resúmenes. 
Figura 3. Categorías centrales detectadas

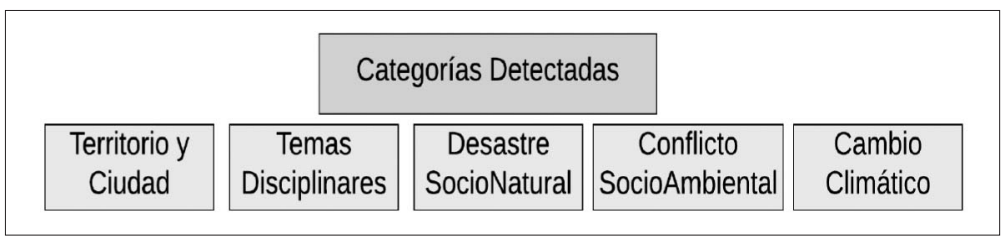

Fuente: elaboración propia.

La categoría "Territorio y ciudad" agrupa la mayor cantidad de ponencias a partir de temas que -con foco puesto en el desarrollo sostenible- incorporan elementos asociados en el engranaje entre la vida urbana, el despliegue comunitario y problemáticas socioecológicas.

En la figura 4 se presenta la prevalencia de los resúmenes según cada categoría central detectada.

Figura 4. Distribución de los resúmenes según categorías.

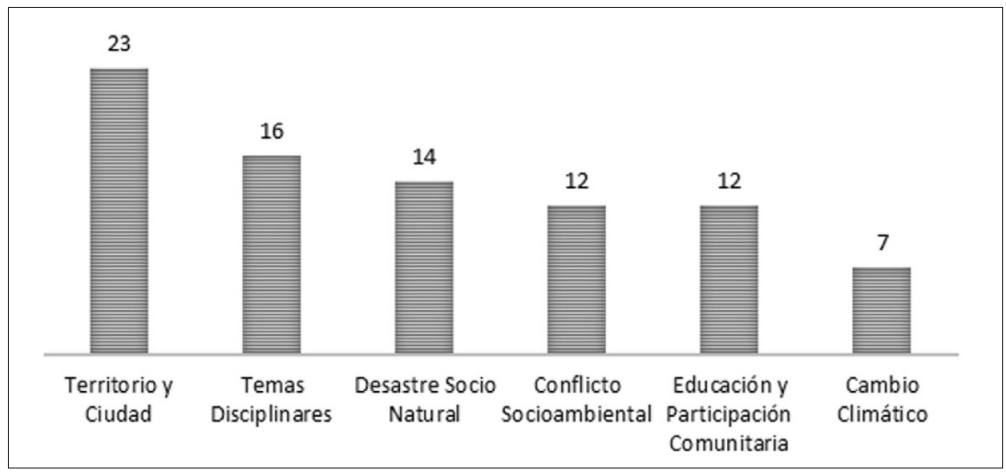

Fuente: elaboración propia.

Por tratarse de un Congreso específico de trabajo social, los temas disciplinares son transversales a todas las ponencias. Sin embargo, se detectaron 16 resúmenes que centran su argumento en el papel del trabajo social respecto de los procesos de formación disciplinar e intervención social frente a las problemáticas ambientales.

El contexto problemático, que surge desde el impacto del cambio ambiental global en las sociedades (Duarte et al., 2006), se manifiesta en resúmenes que relevan y visibilizan las situaciones de 
Trabajo social en el campo socioambiental: discusiones, énfasis y desafíos a partir de la Conferencia Mundial de Trabajo Social 2018 /Sepúlveda

conflicto. A través de dichas comunicaciones se da cuenta de disputas y nudos críticos que se expresan en diversas problemáticas que afectan tanto a las personas como al patrimonio natural, a través de la cuales se expresan injusticias ambientales que afectan a comunidades locales, pero también al sistema global.

Por otra parte, si bien las categorías de desastre socionatural y cambio climático tienen una relación de causa efecto muy estrecha, se abordan desde la especificidad social de cada uno y son los fenómenos con componente natural (no solo antrópico) más mencionadas. En este sentido, se argumenta la emergencia de la cuestión ambiental a partir de las crecientes evidencias científicas respecto del aumento de los desastres naturales y las variaciones del clima de origen antrópico.

Otra categoría detectada se refiere a la educación y la participación comunitaria. Es un aspecto que se menciona constantemente como estrategia metodológica, ética y práctica a la hora de abordar el tópico socioambiental.

La educación ambiental en específico y las resiliencias y buenas prácticas comunitarias ocupan un lugar central dentro del debate. En estos resúmenes, el énfasis está puesto en el relato de factores protectores compartidos a la hora de enfrentar los conflictos o injusticias ambientales. Destaca en esta categoría un reconocimiento de las resiliencias que poseen los pueblos indígenas y se hace énfasis en la necesidad de visibilizar los saberes locales y ancestrales disponibles en el contexto de intervención o investigación. 


\section{d) Temas específicos según categorías detectadas.}

Para ordenar y sintetizar la información, en la tabla 1 se presenta un resumen de cada tema específico según categoría. En el lado izquierdo de la tabla se menciona la categoría específica detectada y a la derecha una síntesis de los temas, conceptos y problemáticas que componen el debate, y que dan cuenta de la diversidad y riqueza de la discusión.

Tabla 1: Temas específicos según categorías detectadas

\begin{tabular}{|c|c|}
\hline Categoría & Temas específicos \\
\hline $\begin{array}{l}\text { Temas } \\
\text { disciplinares }\end{array}$ & $\begin{array}{l}\text { - Discusión sobre el concepto de "Antropoceno". } \\
\text { - Papel del Trabajo Social, TS en: 1) La construcción de } \\
\text { sociedades más sustentables, 2) Las políticas de ahorro } \\
\text { de recursos naturales y 3) Las estrategias de mitigación } \\
\text { y adaptación frente a los desastres o conflictos socioam- } \\
\text { bientales. } \\
\text { - Ética ambiental y bioética. } \\
\text { - Roles, responsabilidades y acciones de trabajadores y tra- } \\
\text { bajadoras sociales en lo ecosocial. } \\
\text { - Estado de la investigación de TS. } \\
\text { - Papel de los académicos de TS en la formación de estu- } \\
\text { diantes con conciencia ambiental. } \\
\text { - Procesos y desafíos en proyectos de trabajo ecosocial. } \\
\text { - Incorporación de la temática en los planes de estudio de } \\
\text { TS. }\end{array}$ \\
\hline $\begin{array}{l}\text { Territorio y } \\
\text { ciudad }\end{array}$ & $\begin{array}{l}\text { - Planificación urbana y acceso a los espacios públicos. } \\
\text { - Buenas prácticas para construir paisajes urbanos soste- } \\
\text { - Soberanía alimentaria y comercio justo. } \\
\text { - Gentrificación verde. } \\
\text { - Gobernanza ambiental. } \\
\text { - Gestión de residuos. } \\
\text { - Planes locales de sostenibilidad. } \\
\text { - Morfología urbana, asentamientos y vulnerabilidad. } \\
\text { - Crecimiento demográfico. } \\
\text { - Bioregionalismo y sistemas ecológicos integrados. } \\
\text { - Calidad del aire y bienestar subjetivo. } \\
\text { - Innovaciones sociales y económicas hacia un desarrollo } \\
\text { - Sostenible. }\end{array}$ \\
\hline
\end{tabular}


Trabajo social en el campo socioambiental: discusiones, énfasis y desafíos a partir de la Conferencia Mundial de Trabajo Social 2018 /Sepúlveda

\begin{tabular}{|c|c|}
\hline $\begin{array}{l}\text { Cambio climático } \\
\text { (en adelante CC) }\end{array}$ & $\begin{array}{l}\text { - Percepción comunitaria y local del CC. } \\
\text { - Seguridad alimentaria y CC. } \\
\text { - Planificación comunitaria frente a los impactos del CC en } \\
\text { los recursos hídricos. } \\
\text { - Agricultura, pesca artesanal y CC: impactos y estrategias } \\
\text { de adaptación. } \\
\text { - Acciones y relaciones comunitarias para enfrentar el CC. } \\
\text { - Impacto del CC en las mujeres. } \\
\text { - Discusión disciplinar del CC como problemática social. }\end{array}$ \\
\hline $\begin{array}{l}\text { Desastre } \\
\text { Socionatural (en } \\
\text { adelante DSN) }\end{array}$ & $\begin{array}{l}\text { - Resiliencias comunitarias frente a DSN. } \\
\text { - Gestión del riesgo de desastres. } \\
\text { - Enfoques de intervención. } \\
\text { - Resiliencia infantil frente a DSN. } \\
\text { - Capitalismo y DSN. } \\
\text { - Estrategias de reconstrucción social posdesastre. } \\
\text { - Asistencia social y financiera posdesastre. } \\
\text { - Respuesta del TS frente a un DSN. } \\
\text { - Impacto en la salud mental. } \\
\text { - Impacto en los pueblos indígenas. } \\
\text { - Investigación del TS en el tema. }\end{array}$ \\
\hline $\begin{array}{l}\text { Conflictos so- } \\
\text { cioambientales }\end{array}$ & $\begin{array}{l}\text { - Impactos sociales de la crisis ambiental. } \\
\text { - Desigualdad en el acceso al agua. } \\
\text { - Pobreza energética. } \\
\text { - El problema del hambre en el mundo. } \\
\text { - Riesgos ecológicos comunitarios. } \\
\text { - Migración ambiental y desplazados ambientales. } \\
\text { - Injusticia ambiental. } \\
\text { - Explotación animal. } \\
\text { - Contaminación ambiental. } \\
\text { - Secreto comercial y prácticas opresivas de las empresas. }\end{array}$ \\
\hline $\begin{array}{l}\text { Educación y par- } \\
\text { ticipación comu- } \\
\text { nitaria }\end{array}$ & $\begin{array}{l}\text { - Educación ambiental. } \\
\text { - Conservación de la biodiversidad. } \\
\text { - Resiliencias comunitarias. } \\
\text { - Activismo ambiental. } \\
\text { - Buenas prácticas ambientales. } \\
\text { - Bienestar sostenible y salud a través de intervenciones } \\
\text { asistidas por la naturaleza. } \\
\text { - Indígenas: buenas prácticas, activismo, resiliencias, estra- } \\
\text { tegias de adaptación. }\end{array}$ \\
\hline
\end{tabular}

Fuente: elaboración propia 


\section{Discusión}

El trabajo social contemporáneo avanza hacia la constitución de un acumulado científico y práctico, en el que la cuestión socioambiental aparece como un asunto de relevancia en el debate disciplinar internacional. El SWSD 2018 marca un hito y es una señal directa en el reconocimiento de la vinculación sociedad y naturaleza como un elemento importante para la comprensión de los contextos en los que se despliega el quehacer profesional.

Lo "social" se compone también de lo "ecosocial", por lo tanto, la profesión transita hacia la construcción de racionalidades ambientales, que cuestionan las posiciones antropocéntricas, economicistas e instrumentales utilizadas para comprender el mundo humano y no humano. En este sentido, los resúmenes dan cuenta de un saber ambiental disciplinar que abre las fronteras de las perspectivas rígidas y propone representaciones, técnicas y prácticas que se apropian de la vinculación socioambiental (Leff, 2004). En consecuencia, surge un "trabajo social verde", un "trabajo ecosocial", un "trabajo social ambiental" que abre las puertas a un rico debate teórico, metodológico y epistemológico.

Generalmente, la identidad del trabajo social se ha sostenido en dispositivos anclados a la intervención social a partir de una profesión eminentemente práctica y con orientación tecnológica (Yañez, 2016). Aquello ha sido la tónica del trabajo social latinoamericano, por ejemplo, lo que se plasma en el histórico debate respecto a la conciliación entre teoría y praxis, entre intervención y ciencia.

Pese a ello, la mirada mundial que permite la revisión de los resúmenes visibiliza nuevos protagonismos, esta vez vinculados a la investigación social y el conocimiento científico. De hecho, más de la mitad de los resúmenes revisados son resultados de procesos de investigación.

Dicho conocimiento se crea y constituye desde los saberes y recursos de las ciencias sociales, en diálogo con las trayectorias dis- 
Trabajo social en el campo socioambiental: discusiones, énfasis y desafíos a partir de la Conferencia Mundial de Trabajo Social 2018 /Sepúlveda

ciplinares del trabajo social. Sin duda, es un avance importante en la tarea de fortalecer la dimensión científica de la profesión.

Sin embargo, se evidencia una desigualdad espacial innegable en la generación y socialización del conocimiento científico que se produce. Los países del sur del mundo, generalmente en condición de pobreza y con políticas débiles de apoyo a la ciencia y la investigación, tienen una presencia muy escasa en el debate.

Esto permite visibilizar una paradoja, ya que son justamente los países del sur del mundo quienes presentan mayores niveles de vulnerabilidad frente a desastres naturales, además de deficiencias profundas en las estrategias de adaptación y gobernanza frente a conflictos o catástrofes socioambientales (Ecktein y Melchior, 2017; Bundnis Entwicklung Hilft, 2017).

De hecho, si bien los temas específicos detectados (tabla 1) son diversos y fundamentales, no se mencionan problemáticas ambientales características del sur-sur. Por ejemplo: investigaciones sobre los movimientos sociales y campesinos de defensa del derecho a la tierra y el agua; violencia empresarial y estatal hacia las comunidades que resisten la instalación de megaproyectos extractivistas; seguridad de los dirigentes medioambientales; conflictos armados y, por último, colonialismo y naturaleza, entre otros.

Esta situación plantea un desafío al trabajo social en Chile y Latinoamérica. De lo que se trata es de aumentar las investigaciones y los análisis vinculados a la cuestión socioambiental, así como también marcar presencia internacional en las diversas instancias de diálogo interdisciplinar académico. Por otra parte, el debate está marcado por dos aspectos que se expresan de manera permanente en los resúmenes analizados. En primer lugar, la relevancia de la dimensión ética de la acción. La crisis ambiental y sus causas requieren de narrativas que inyecten a la acción elementos éticos que enmarquen y orienten la crisis. Dichas orientaciones provienen generalmente de los acuerdos internacionales, como la Declaración Universal de los Derechos Humanos, el Marco de Sendai y los Objetivos de desarrollo sos- 
tenible. La justicia ambiental es el principio ético cardinal que busca construir entornos y contextos respetuosos con los sistemas socioecológicos, y así avanzar hacia una economía solidaria de sustento.

En segundo lugar, las autoras y autores otorgan relevancia a un trabajo social que promueva las resiliencias y saberes locales. Mencionan que trabajadores y trabajadoras sociales deben aprender y aplicar los saberes ancestrales o tradicionales de las comunidades, con foco en la ayuda mutua y los procesos compartidos. Se trata de un trabajo social con sello intercultural y comunitario, capaz de ajustarse a la diversidad socioecológica de cada territorio.

En este sentido, el debate disciplinar concuerda en que la acción profesional debe fortalecer el activismo de base, los modelos económicos alternativos y una crítica sostenida al capitalismo y su modelo masivo de consumismo industrial.

Si bien se reconocen brechas en cuanto a la exploración, teorización o aplicación pragmática del trabajo social ambiental como subcampo disciplinar, existe un acumulado de investigaciones, experiencias y herramientas de alto valor disciplinar y contextual.

\section{Conclusiones}

La tribuna que el SWSD 2018 le otorgó al desarrollo sostenible y a la vinculación socioambiental, visibiliza un trabajo social poseedor de trayectorias, procesos y recursos que permiten dar respuestas efectivas a los desafíos contemporáneos que acompañan el cambio ambiental global. El debate estuvo centrado en la construcción de sistemas éticos y sociopolíticos para forjar sociedades que tomen conciencia y actúen frente a las problemáticas sociales y ambientales contemporáneas, especialmente las asociadas a la vida urbana. Una vida urbana cuya morfología evidencia vulnerabilidades, riesgos y desigualdades que requieren de un trabajo social que -desde la investigación y la intervención- active gestiones e innovaciones para la creación de sistemas que integren lo ecológico, lo cultural y lo social. 
Trabajo social en el campo socioambiental: discusiones, énfasis y desafíos a partir de la Conferencia Mundial de Trabajo Social 2018 /Sepúlveda

Se hace énfasis en reconocer que el desarrollo sustentable es un valor necesario e ineludible a la hora de pensar el bienestar y la calidad de vida de los territorios. Los procesos de planificación urbana y uso de los espacios públicos es un desafío para la profesión, especialmente en lo referido a integrar la voz y capitales socioculturales de las comunidades en las decisiones y acuerdos.

Por otra parte, los desastres naturales y el cambio climático son fenómenos que también destacan en el debate. Dichos fenómenos han sido conceptualizados y comprendidos generalmente por científicos y académicos de las ciencias de la tierra a partir de miradas que enfatizan en los elementos geográficos, biológicos o naturales.

Sin embargo, las autoras de las ponencias hacen énfasis en el cambio climático como un desafío político mundial, que deja entrever las posiciones ideológicas, económicas y éticas a escala global. A su vez, los desastres naturales son cada vez menos naturales y la variable antrópica acelera procesos de adaptación y mitigación de riesgos en los que las resiliencias comunitarias y los saberes locales son centrales.

Los conflictos socioambientales que caracterizan el Antropoceno incorporan en la agenda pública a movimientos y demandas sociales que ponen en jaque las promesas del capitalismo y el progreso extractivista. Frente a ello, la mirada sociocultural, comprensiva y analítica del trabajo social respecto de estos fenómenos, aporta narrativas y perspectivas que enriquecen las miradas integrales e interdisciplinarias que requiere la realidad compleja.

Hacia la búsqueda de nuevas ecologías de los saberes, de economías sustentables (Rawoth, 2017) y de nuevas formas de entender nuestra posición en la red compleja de la vida (Margulis, 1995), el SWSD 2018 permite proyectar rutas de acción y énfasis que desafían a las y los trabajadores en tres aspectos centrales:

1) Reconocimiento de la relevancia de la vinculación ambiental y el desarrollo sostenible como un tema clave que incide en la 
vida de las personas, familias y comunidades, especialmente aquellas expuestas a guerras, cambio climático y altos grados de desconfianza. Es un tema que está en el corazón de los principios y valores del trabajo social, en donde se deben volcar las habilidades, conocimientos y experiencias que ha acumulado la profesión.

2) Aumentar la investigación asociada a temáticas socioambientales. Se enfatiza la importancia de consolidar redes de colaboración entre centros/núcleos de investigación y universidades de contextos diversos.

3) Fortalecer procesos de formación profesional que integren temáticas socioecológicas a partir de elementos relacionados con la justicia ambiental, el trabajo comunitario y el activismo ambiental. Se enfatiza la importancia de la conciencia y sensibilización de las cuestiones socioambientales en los y las estudiantes de Trabajo Social, así como también en los colectivos profesionales.

\section{Bibliografía}

Arias, M. (2018). Antropoceno, La política en la era humana. Barcelona, España: Editorial Taurus.

Bundnis Entwicklung Hilft. (2017). World Risk Report 2017 Analysis and prospects. Recuperado de http://weltrisikobericht.de/wpcontent/uploads/2017/11/WRB2017_online3.pdf

Caidolls, F. (2013). Regional divides in global environmental change research capacity Introduction to Part 2, in Word Social Science Report Changing Global Environments (pp. 125132). Paris, Francia: UNESCO, ISSC, CLACSO.

Duarte, C., Alonso, S., Benito, G., Dachs, J., Montes, C., Pardo, M., Ríos, A., Simó, R. y Valladares, F. (2006). Cambio Global. Impacto de la Actividad Humana sobre el Sistema Tierra. Madrid, España: Consejo Superior de Investigaciones científicas (CSIC).

IASSW AIETS/ ICSW. (2012). The Global Agenda For Social Work and social development commitment to action. Recuperado de https://www.ifsw.org/wpcontent/uploads/ifswcdn/assets/ globalagenda2012.pdf. 
Trabajo social en el campo socioambiental: discusiones, énfasis y desafíos a partir de la Conferencia Mundial de Trabajo Social 2018 /Sepúlveda

IASSW/ICSW/IFSW. (2018). Abstract Book SWDS 2018. Recuperado de https://www.swsd2018.org/wpcontent/uploads/2018/07/ SWSD2018_Abstract-Book.pdf.

Kreft, S., Ecktein, D. y Melchior, I. (2017). Global Climate Risk Index 2017. Recuperado de https://germanwatch.org/en/12978.

Leff, E. (2004). La Racionalidad ambiental. La reapropiación social de la naturaleza. Buenos Aires, Argentina: Siglo XXI.

Margulis, L. y Sagan, C. (1995). Microcosmos. Recuperado de https://doi. org/10.2307/1310716

Martínez Alier, J. (2001). Economía ecológica y política ambiental. Ciudad de México, México: Fondo de Cultura Económica.

Miller, S. y Hayward, R. (2014). Social work education's role in addressing people and a planet at risk. Social Work Education, 33(3), 280-295.

Naredo, J. M. (2006). Raíces económicas del deterioro ecológico y social: más allá de los dogmas. Madrid, España: Siglo XXI.

Ramsay, S \& Boddy, J. (2017). Environmental Social Work: A Concept Analysis. The British Journal of Social Work, 47(1), 68-86. Recuperado de https://doi.org/10.1093/bjsw/bcw078.

Raworth, K. (2017). Doughnut Economics. USA: Chelsea Green.

Truell, R., \& Jones, D. (2017, March 29). Global Agenda for Social Work and Social Development. Encyclopedia of Social Work. Ed. Recuperado de http://oxfordre.com/socialwork/view/10.1093/acrefore/9780199975839.001.0001/ acrefore-9780199975839-e-1158.

United Nations/UNISDR. (2015). Global Assessment report on disaster risk reduction. Recuperado de https://www.preventionweb.net/english/hyogo/gar/2015/en/home/GAR_2015/ GAR_2015_2.html.

Wallemacq, P., Below R. y McLean D. (2018). Econimics Loses, poverty \& disaster. Recuperado de https://www.emdat.be/publications.

Yañez, V. (2016). Notas sobre la cosmología disciplinar del trabajo social. Tabula Rasa. Bogotá, Colombia, (25), 415-427. 